\title{
Precision medicine in kidney disease: the patient's view
}

\section{Keith D. Brown, Catherine Campbell and Glenda V. Roberts}

Abstract | Research in the field of nephrology continues to improve our understanding of the mechanisms that promote and drive kidney disease, including how human genetic variation might affect disease predisposition and progression. One of the goals of these research efforts is to inform and enable the implementation of precision medicine, whereby patient management is tailored to the individual according to the mechanisms underlying their disease to increase the chances of therapeutic success. To achieve this goal, we need a clearer understanding of the molecular pathways that underlie the many different causes of kidney failure. These research insights are being increasingly translated and implemented into clinical practice. In this Viewpoint, we asked three individuals who have been affected by kidney failure for their views on the importance of understanding the drivers of kidney disease and, on a personal level, what they hope might be achieved with this information.

\section{Q. Have advances in our understanding of the molecular basis of kidney disease affected the management of your disease?}

Keith D. Brown. I had a successful kidney transplant 10 years ago and have been doing well. Prior to my transplant, I underwent a percutaneous kidney biopsy to determine the type and extent of my kidney disease. Because my transplant has been functioning so well, there hasn't been a direct correlation between the management of my transplant and advances in our understanding of the molecular basis of kidney disease. I'm fortunate that my nephrologist, who has a limited clinical practice, is highly involved in kidney research. In the event that my focal segmental glomerulosclerosis should ever return, I'm hopeful that advances in our understanding of the molecular basis of kidney disease will mean that information will have a much larger role in the management of the disease than it might have had 10-15 years ago.

Catherine Campbell. I am privileged to have had a kidney biopsy as this procedure is not always routinely available to patients with kidney disease. Being aware of my biopsy findings, which included the presence of fibrotic scarring commonly found in chronic kidney disease (CKD), has helped me understand better the causes behind my kidney failure and has empowered me to manage my self-care. These findings have improved my desire to slow the disease by watching my diet and blood glucose levels, which helps me manage my diabetes, as well as monitoring my blood pressure, avoiding potential falls and resting to avoid fatigue. I have accomplished these goals successfully through medication compliance and regular lab draws and self-injections of an erythropoiesis-stimulating agent. Knowing the molecular basis of my disease provides a foundational base for my care and the care of others. As a Doctor of Nursing Practice, I believe it is within my personal and professional responsibilities to educate patients, families and the community of ways to prevent $\mathrm{CKD}$.

Glenda V. Roberts. Although advances in our understanding of the molecular basis of kidney disease have not affected the management of my disease because I have a transplant, I believe that they are beginning to affect the management of kidney disease for patients who do not have transplants. For instance, the study of biopsy samples from patients with acute kidney injury (AKI) and CKD is beginning to expand our understanding of the underlying causes of these conditions and, in some cases, to improve diagnostics. These discoveries are being shared within research institutions. Clinical research discoveries are likely to be more rapidly adopted by physicians involved in clinical research, who are often exposed to the research findings during Renal Grand Rounds or other academic meetings, often before those findings are published. Often it takes years for clinical research findings to be adopted by physicians whose practice is outside of the university research environment. I think that patients who are managed by nephrologists who work at research institutions will probably be the most immediately affected because their physicians are exposed to the discoveries and insights that their research peers are developing. As researchers start to publish more of their discoveries, I expect to see more patients benefit from this enhanced molecular understanding. It is my hope that the inclusion of a 'lay abstract' in medical research articles will become a standard requirement by publishers. Such abstracts would enable patients to understand the reported research findings and empower them to ask questions and discuss the research with their physicians, which would likely impact the management of their disease. We know that global kidney scarring is more pronounced in patients with late-stage disease than in those with early-stage disease. Therefore, early-stage biopsies, coupled with an improved understanding of the molecular basis of kidney disease, might make it easier for pathologists and nephrologists to assess the potential causes of disease and its progression, which would likely influence how the patient's disease is managed.

How important is it for you to understand the underlying mechanistic basis of your disease?

K.D.B. The human body is a magnificent thing and I have always had an interest in human physiology. Therefore, I have always been very interested in the pathophysiology and disease mechanism of kidney disease. When your own kidneys are failing, you 
quickly learn all the various regulatory functions that the kidney performs.

C.C. Identifying the underlying mechanisms of my kidney disease has been a revolutionizing experience. I had always been somewhat focused on my blood sugars and blood pressure, but the results of my kidney biopsy relayed a surprise finding although I have diabetes, the main cause of my kidney disease is hypertension. Also, as a child I had frequent urinary tract infections (UTIs), but a potential cause for their recurrence was never investigated. Urinary incontinence has also been a lifetime challenge and embarrassment. In kindergarten I was punished when having to go to the bathroom during class. I was also punished by a family member for wetting on the way home from school, even though I had been to the bathroom before leaving. These growing pains were emotionally painful and humiliating. However, my biopsy results gave me some insight into these events, as the presence of fibrotic tissue is likely linked to complications from frequent UTIs that resulted in kidney infections ${ }^{1}$. Participating in the Kidney Precision Medicine Project (KPMP) consortium as a patient with CKD and being involved in community engagement and education have provided me with a basic understanding of the origins of my CKD - I have a family history of type 2 diabetes and hypertension, which are heritable risk factors for CKD. Although I cannot change the effects that these risk factors have had on my health, my improved understanding of the disease basics has educated and improved my self-care.

\section{G.V.R. It is very important to me to} understand the underlying mechanistic basis for my disease because it enables me to take informed action. For instance, if I know that eating too much kale increases the likelihood of developing kidney stones and I have a propensity towards kidney stone development, then I can change my diet accordingly.

What do you envisage precision medicine will mean for the diagnosis, prognosis and treatment of kidney disease in the future? What are your hopes for the short and long-term?

K.D.B. I hope that new discoveries in precision medicine will, in the short-term, lead to treatments that will significantly delay the progression of kidney disease. Once we find ways to better understand what cellular or molecular or genetic attributes are related to an individual's kidney disease, then treatments can be developed to modify or suppress these various causes of the disease. Delaying the progression of kidney disease will hopefully reduce the need for dialysis and

\section{The contributors}

Keith D. Brown is a NIH Kidney Precision Medicine Project (KPMP) Patient Participant and a member of the Steering Committee and Community Engagement Committee. He received his law degree from Gonzaga University in Spokane, Washington, in 1985 and has practised in the area of medical malpractice defence for over 30 years. He served on the Providence Institutional Review Board-Spokane from 2007 to 2019.

Catherine Campbell has been a registered nurse for more than 40 years. Her life purpose is committed to educating and servicing others in health concerns. She has made multiple contributions in volunteer service: as a Patient Participant in the KPMP, an American Association for Retired Persons (AARP) public policy advocate, a Board of Directors member for the Case Management Society of America, a Life Fellows advocate in the American College of Healthcare Executives, Dallas/Fort Worth Community Advisory Board for kidney patient support, an American Association of Kidney Patient advocate and a Governance Committee member for the Sigma Nu Upsilon chapter. In her continued commitment to the nursing profession, she mentors careerists in the American College of Healthcare Executives, as well as doctoral and master nursing students, and delivers online teaching to nursing students attending Mount Saint Mary's University, Los Angeles.

Glenda V. Roberts is the Director of External Relations \& Patient Engagement at the University of Washington Center for Dialysis Innovation (CDI) and the Kidney Research Institute (KRI). She brings the patient voice to a number of NIH/NIDDK government and industry research efforts, as well as the American Society of Nephrology (ASN) COVID-19 Response Team and the ASN COVID-19 Transplant Subcommittee. She is a member of the International Society of Nephrology (ISN), the Kidney Health Initiative (KHI) Patient and Family Partnership Council (PFPC), Can-SOLVE CKD International Research Advisory Committee and Home Dialyzors United Advisory Board, and has been an Ambassador for the American Association of Kidney Patients since 2018. In addition to being a Patient Participant, Glenda is a member of the Steering Committee, the Collaborations Committee, the Return of Results Committee and the Community Engagement Committee, as well as the Director of Communications, for the KPMP.

kidney transplants in the short term. In the long term, I think the increased scientific knowledge we gain will lead to an eventual cure for kidney disease. Advances are sure to come in genetic engineering and new medications that will correct whatever is causing an individual's kidney disease.

C.C. The current limited treatment options can be devastating for patients with CKD.

Advances in precision medicine can only be viewed as positive for these patients. They are also vital to the families of patients with CKD to ensure early diagnosis and treatment in the future. For example, the presence of CKD risk factors with a strong genetic component, such as type 2 diabetes, hypertension or heart disease in my family, might increase the likelihood that another family member might develop CKD. Current research efforts include the KPMP, which aims to study kidney biopsy samples to develop a kidney atlas in which molecular information is catalogued to help identify the underlying causes of kidney disease. I hope that this project will lead to improved early disease diagnosis and specific treatments, as well as providing vital education for physicians, patients and their families. The Community Advisory Board includes members from the community who have been selected to include a diverse population to facilitate community inclusion in education and research, for example, by recruiting individuals to provide biopsy tissue, and to enable health recommendations to reach the under-served and those with predisposition to kidney disease. Biopsy samples are currently being collected from patients with $\mathrm{AKI}$ or CKD and my long-term vision for maximizing the potential of these resources includes not only the continued procurement of biopsies but also the collection of biopsy samples from healthy individuals for comparison with those from individuals with kidney disease. I envision community-wide understanding and participation in rallying for the development of, and access to, appropriate treatments. Today, Medicare only provides 3 years of anti-rejection drugs to transplant recipients, which disproportionally affects and limits the life expectancy of patients who cannot afford these medications independently. In the long term, patients and their families must be informed and empowered to speak up for their right to access appropriate treatment regimens.

G.V.R. I hope that in the future, it will be standard of care to recommend a kidney biopsy to anyone who is diagnosed with kidney disease. Considering the diagnostic 
challenges resulting from the increase in scarring as kidney disease progresses, by identifying the cause of an individual's kidney disease earlier, we will hopefully be able to implement a treatment tailored for that individual to slow or reverse the progress of their kidney disease. Most doctors do not recommend biopsies in the early stages - they tend to wait until a patient is stage 3 or later - and one explanation is that a biopsy is surgery and thus discouraged. I hope that as doctors become exposed to the things that we're learning in the KPMP they'll appreciate the benefit of a biopsy. In the short-term, I hope that as many patients as possible will continue to participate in research projects, for example by signing up to get biopsies and contribute to the KPMP kidney atlas, so that researchers gain better insight into the cells and pathways that cause various diseases. This atlas will enable us to better divide the causes of kidney disease into subgroups and identify the appropriate treatment for each subgroup, rather than the one-size-fits-all approach that is used today. Historically, diseases like cancer and kidney disease were treated with a one-size-fits-all approach. We see that the cancer research field is making good progress in terms of developing more personalized treatments, and the outcomes are encouraging. I am looking forward to the development of similar novel therapies and treatments for people with kidney disease.

\section{Can you see any drawbacks to a precision medicine approach to kidney} disease? Is there likely to be an impact on family members?

K.D.B. In my view, more scientific knowledge and evidence-based medicine are always better than less. However, one drawback when we're dealing with genetic testing is, of course, the concern about the privacy of an individual's genetic information. In a worst-case scenario, a person has to worry about things like insurability and employability if certain genetic information is revealed. Even things like whether or not to have children can be an issue if you are likely to pass on a gene that increases the risk of kidney disease in your offspring. As physicians and scientists learn more about the specific genetic and molecular characteristics of an individual that are causing or contributing to their kidney disease, this will likely have an impact on their family members. For example, family members who might have inherited these genetic predispositions to kidney disease will be faced with decisions about whether or not they want to undergo genetic testing. As with certain genetic variants that are related to certain cancers, some family members will want to get tested and begin whatever individualized treatment might be appropriate; others might prefer not to know whether they are predisposed to developing kidney disease.

C.C. I do not foresee any drawbacks to precision medicine. Genetics and personalized treatments are already recognized in other diseases. For example, regular mammography might be recommended for early detection of breast cancer in individuals known to have inherited genetic variants that research has identified as being associated with an increased likelihood of developing breast cancer. Such hereditary risk variants might also be identified in the context of kidney disease. Breast cancer research has also demonstrated, for example, associations between different lifestyles and health status, and the risk of developing breast cancer ${ }^{2}$. It might be possible to obtain the same type of information about the development of CKD by advancing research through the study of kidney biopsy samples. Recognizing the potential risks in a family might prevent children from developing CKD through education and information, which are vital to the future of our young and might enable them to improve their lifestyle and minimize the risks to their health. I might have benefited from this information at a younger age - there is no guarantee that this support would have decreased my kidney failure, but hopefully precision medicine will still help improve the lasting poor prognosis.
G.V.R. I do not see any drawback to precision medicine, per se. However, based on some historical practices by insurance companies, I think that some people will be reluctant to get a personalized diagnosis because it might be viewed as a pre-existing condition. I think that this is a legitimate concern. The question becomes whether the individual gets treatment now to slow or reverse the condition or whether they wait and let the disease put them at greater risk in order to get insurance coverage. Hopefully, someday people will not have to worry about their family history or pre-existing conditions preventing them from getting insurance coverage.

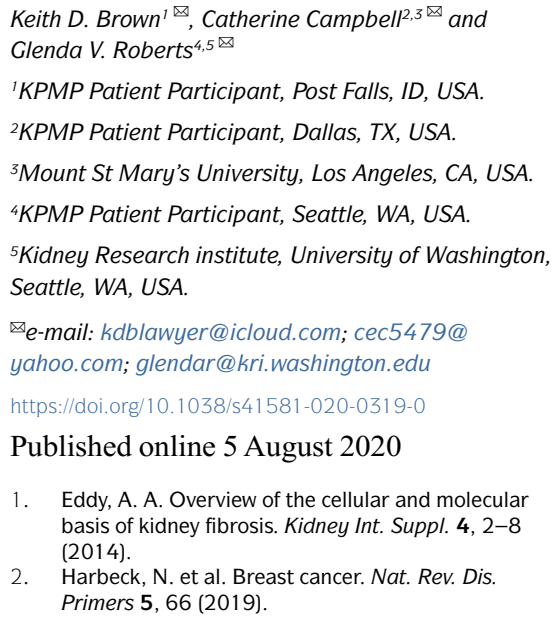

Published online 5 August 2020

1. Eddy, A. A. Overview of the cellular and molecula basis of kidney fibrosis. Kidney Int. Suppl. 4, 2-8 (2014).

2. Harbeck, N. et al. Breast cancer. Nat. Rev. Dis. Primers 5, 66 (2019).

\section{Acknowledgements}

The KPMP is funded by the following grants from the NIDDK: U2C DK114886, UH3DK114861, UH3DK114866, UH3DK 114870 , UH3DK 114908 , UH3DK 114915 , UH3DK 114926, UH3DK 114907 , UH3DK 114920 , UH3DK114923, UH3DK114933 and UH3DK114937. The content is solely the responsibility of the authors and does not necessarily represent the official views of the National Institutes of Health.

\section{Competing interests}

G.V.R. is employed by the University of Washington, has contributed to the National Palliative Care Grant (patient advisor) and has participated in the following NIH-funded projects: KPMP (Director of Communications), APOLLO (Community Advisory Committee member) and APOL 1 (Community Advisory Board member). K.D.B. and C.C. declare no competing interests.

\section{Publisher's note}

Springer Nature remains neutral with regard to jurisdictional claims in published maps and institutional affiliations.

(C) Springer Nature Limited 2020 